\title{
ATIVIDADE FÍSICA COMUNITÁRIA: EFEITOS SOBRE A FUNCIONALIDADE NA LOMBALGIA CRÔNICA
}

\author{
Community physical activity: effects on functionality in chronic low back pain \\ Actividad física en la comunidad: efectos sobre la funcionalidad en la lumbalgia crónica
}

\author{
Maicon da Silva Martins \\ Universidade do Extremo Sul Catarinense - UNESC - Santa Catarina (SC) - Brasil \\ Willians Cassiano Longen \\ Universidade do Extremo Sul Catarinense - UNESC - Santa Catarina (SC) - Brasil
}

\section{RESUMO}

Objetivo: Avaliar a intensidade dolorosa, a força lombar e a funcionalidade de indivíduos que possuem lombalgia crônica inespecífica e são praticantes de um programa públicos de exercícios físicos. Métodos: Pesquisa exploratória, transversal e quantitativa realizada em 2017, com 130 mulheres de 30 a 60 anos de idade e sem histórico de cirurgia na coluna vertebral. Aplicou-se o Oswestry Low Back Pain Disability Questionnaire (ODQ) para a avaliação da incapacidade relacionada à coluna lombar. Quantificou-se a dor através da escala visual analógica (EVA) e a força muscular lombar com dinamometria lombar (Takei $\left.{ }^{\circledR}\right)$. Resultados: Das 130 participantes, 97,7\% ( $\mathrm{n}=127$ ) apresentaram incapacidade funcional mínima relacionada à coluna lombar (ODQ). Em relação à força, 16,9\% (n=10) apresentaram valores normais e $83,1 \%(n=120)$ abaixo dos valores de referência, apresentando fraqueza muscular. Quanto à intensidade da dor, $47,7 \%(n=67)$ das participantes apresentaram intensidade leve, 45,5\% $(\mathrm{n}=59)$ apresentaram intensidade moderada e $6,9 \%(\mathrm{n}=9)$, intensa. Conclusão: $\mathrm{O}$ programa possui características que contribuem para manter controlada a intensidade dolorosa, que vem se mostrando baixa, bem como a incapacidade funcional significativa dos indivíduos. A fraqueza muscular na região lombar, apresentada pelas participantes sem associação com a funcionalidade, e a dor podem ser um fator que perpetua os quadros de lombalgia crônica. Os achados sugerem a necessidade de revisão da prescrição terapêutica dos exercícios do programa realizado.

Descritores: Dor Lombar; Promoção da Saúde; Exercício.

\section{ABSTRACT}

Objective: To evaluate the pain intensity, lumbar strength and functionality of individuals presenting chronic non-specific low back pain and taking part in a public physical exercise program. Methods: Exploratory, cross-sectional and quantitative survey conducted in 2017 with 130 women from 30 to 60 years of age and without history of spinal surgery. The Oswestry Low Back Pain Disability Questionnaire (ODQ) was applied for assessment of the disability related to the lumbar spine. The pain was quantified through the visual analog scale (VAS) and the lumbar muscle strength through lumbar dynamometry (Takei $(\mathbb{R})$. Results: Of the 130 participants, 97.7\% ( $n=127)$ presented minimal functional disability related to the lumbar spine $(O D Q)$. In respect to strength, $16.9 \%(n=10)$ presented normal values and $83.1 \%(n=120)$ were below the reference values, presenting muscle weakness. As for the pain intensity, $47.7 \%(n=67)$ of the participants showed mild intensity, $45.5 \%(n=59)$ presented moderate intensity and $6.9 \%(n=9)$, severe. Conclusion: The program has characteristics that contribute to maintaining under control the pain intensity, which has been slight, as well as the significant functional disability of the individuals. The muscle weakness in the lumbar region, presented by the participants without association with functionality, and the pain as well, might be factors that perpetuate the chronic low back pain. The findings suggest the need to revise the therapeutic prescription of the exercises of the implemented program.

Descriptors: Low Back Pain; Health Promotion; Exercise. 


\section{RESUMEN}

Objetivo: Evaluar la intensidad del dolor, la fuerza de la región lumbar y la funcionalidad de individuos que tienen lumbalgia crónica inespecifica y que participan de un programa público de ejercicios físicos. Métodos: Investigación exploratoria, transversal y cuantitativa realizada en 2017 con 130 mujeres entre 30 y 60 años de edad y sin histórico de cirugía de la columna vertebral. Se aplicó el Oswestry Low Back Pain Disability Questionnaire (ODQ) para evaluar la incapacidad relacionada con la región lumbar. Se cuantificó el dolor a través de la escala visual analógica (EVA) y la fuerza muscular de la región lumbar con la dinamometría (Takei $\left.{ }^{\circledR}\right)$. Resultados: De las 130 participantes, el 97,7\% (n=127) presentaron incapacidad funcional mínima relacionada con la región lumbar (ODQ). Respecto la fuerza el $16,9 \%(n=10)$ presentaron valores normales y el $83,1 \%(n=120)$ presentaron valores por debajo de los de referencia señalando debilidad muscular. Cuanto la intensidad del dolor el 47,7\% $(n=67)$ de las participantes presentaron intensidad leve, el 45,5\% $(n=59)$ intensidad moderada y el 6,9\% (n=9) intensa. Conclusión: El programa tiene características que contribuyen para el mantenimiento de la intensidad del dolor que se presenta baja así como la incapacidad funcional significativa de los individuos. La debilidad muscular de la región lumbar presentada por las participantes sin asociación con la funcionalidad y el dolor pueden ser un factor que se presenta en los cuadros de lumbalgia crónica. Los hallazgos sugieren la necesidad de revisión de la prescripción terapéutica de los ejercicios del programa realizado.

Descriptores: Dolor de la Región Lumbar; Promoción de la Salud; Ejercicio.

\section{INTRODUÇÃO}

O tema promoção da saúde alavancou-se após mudanças políticas no país nos anos 80 , quando o conceito de qualidade de vida começou a ganhar importância, tendo em paralelo a posterior criação da Lei Orgânica da Saúde (1990) e a criação do Sistema Único de Saúde (SUS) que tem como principal objetivo conferir a todos o direito à saúde, sendo o Estado o órgão a dispor de tal benefício ${ }^{(1,2)}$.

A saúde funcional é o estado de funcionalidade e de bem-estar, individual e coletivo, na realização de atividades e na participação social, em que se promove a qualidade de vida e a autonomia para o pleno exercício da cidadania ${ }^{(3)}$. Um importante determinante para a adesão, ou não, em eventos de promoção da saúde está na participação da população ${ }^{(4,5)}$.

Fortes evidências mostram que a inatividade física aumenta o risco de muitas condições adversas à saúde, o que gera uma diminuição da expectativa de vida. A inatividade física é responsável por cerca de 5,3 milhões de mortes no mundo a cada ano, gerando um percentual total de $10 \%$ das mortes entre todas as que acontecem, sendo, portanto, uma grande ameaça à saúde de toda a população ${ }^{(6)}$. A lombalgia está entre os agravos associados à inatividade, como morbidade, com elevado grau de prevalência na população, chegando a $70 \%{ }^{(7,8)}$.

A lombalgia se caracteriza como uma dor na região final da coluna vertebral, entre as últimas costelas e o início da linha glútea. A maioria dos casos pode ser classificada como crônica quando o sintoma persiste por um tempo superior a doze semanas e tem origem inespecífica, não se sabendo qual a sua causa principal ${ }^{(9)}$. Pode estar associada a causas diversas, desde a postura adotada no trabalho e nas atividades laborais até doenças degenerativas e desequilíbrios musculares. Seus principais relatos são aumento de dor ao final do dia por conta da fadiga muscular na região ${ }^{(9,10)}$.

O baixo nível, ou a falta, de atividade física é um dos fatores que podem estar culminando para o aparecimento da lombalgia crônica inespecífica, sendo associado à funcionalidade e/ou desuso da musculatura lombar ${ }^{(11)}$. Na população em geral, a prática de atividade física reduz a dor lombar ${ }^{(12)}$.

Embora a causa específica da maioria dos quadros de lombalgia crônica seja difícil de ser determinada, sabe-se que apresenta associação com o estilo de vida, sendo o sedentarismo, o excesso de peso e a permanência prolongada em certas posições alguns dos fatores considerados de risco potencial ${ }^{(13)}$. A perda da força e da flexibilidade muscular são aspectos relacionados à dor lombar. Esses sintomas são comuns quando o indivíduo apresenta encurtamento dos músculos íliotibiais e isquiotibiais, assim como fraqueza muscular abdominal e de eretores espinhais ${ }^{(14)}$.

Exercícios terapêuticos para a lombalgia podem ser realizados em grupo ou individualmente, contando com vários objetivos físico-funcionais e envolvendo atividade aeróbica, além de fortalecimentos, alongamentos e trabalhos posturais segmentares e globais ${ }^{(15-18)}$

O objetivo do estudo é avaliar a intensidade dolorosa, a força lombar e a funcionalidade de indivíduos que possuem lombalgia crônica inespecífica e são praticantes de um programa públicos de exercícios físicos.

\section{MÉTODOS}

Realizou-se pesquisa exploratória, transversal e quantitativa em centros comunitários da cidade de Criciúma, no estado de Santa Catarina, totalizando 12 bairros, os quais aderem ao programa Ritmo e Saúde, da Associação Feminina de Assistência Social de Criciúma (AFASC). A população envolvida é composta por 400 praticantes distribuídos nas 12 unidades. Após a 
realização de um cálculo amostral, considerando-se erro amostral de $8 \%$ e nível de confiança de 95\%, firmou-se amostraalvo mínima de 110 praticantes. A amostra alcançada totalizou 130 praticantes, com disposição voluntária e consentida de participação no estudo.

A coleta de dados ocorreu nos meses de março e abril de 2017, no período noturno, com auxílio dos bolsistas do Laboratório de Biomecânica (Labiomec) da Universidade do Extremo Sul Catarinense (UNESC). Os bolsistas receberam treinamento envolvendo o emprego da escala visual analógica (EVA) e o posicionamento do dinamômetro lombar, com padronização da avaliação da força e a aplicação do Oswestry Low Back Pain Disability Questionnaire (ODQ).

O Programa Ritmo e Saúde oferece exercícios de força e de resistência muscular, exercícios cinesioterapêuticos realizados em colchonetes, exercícios aeróbicos, como treinamento funcional, caminhadas orientadas e zumba, prescritos por profissionais com o auxílio dos estagiários acadêmicos de Educação Física e de Fisioterapia.

Os critérios de inclusão para a presente pesquisa envolveram mulheres de 30 a 60 anos, participantes do programa há três meses pelo menos, com frequência de, pelo menos, duas vezes por semana nas atividades. Excluíram-se as mulheres com histórico cirúrgico da coluna lombar. Apesar da lombalgia não ser um dos fatores que levam as participantes do presente estudo à adesão ao Programa Ritmo e Saúde, todas as participantes apresentaram dor lombar em algum momento do último ano.

Realizou-se entrevista direta e estruturada e avaliações físico-funcionais da coluna lombar, com instrumentos de incapacidade e de dinamometria lombar, além de entrevista, que apurou dados de identificação, como nome, idade, sexo e profissão.

Para avaliar o nível de capacidade e de incapacidade relacionado à coluna lombar das participantes do programa, aplicouse o Oswestry Low Back Pain Disability Questionnaire (ODQ)(19), que é constituído por 10 sessões e, para cada uma, há seis afirmativas, sendo 5 a pontuação total. Se a primeira afirmativa é eleita pelo respondente, marca-se com pontuação zero (0) e, se for a última, cinco (5) pontos.

A intensidade da dor foi avaliada pela escala visual analógica (EVA), que considera a escala de 0 a 10 , em que zero (0) significa a ausência total de dor e dez (10) é o nível de dor máxima suportável por uma pessoa ${ }^{(20)}$.

Utilizou-se o Questionário Nórdico de Sintomas Osteomusculares (QNSO) para as outras disfunções corporais. O QNSO é constituído por um mapa corporal subdividido em regiões do corpo humano: pescoço; ombros; cotovelos; antebraços; punhos; mãos e dedos; região dorsal; região lombar; quadris e/ou coxas; joelhos; tornozelos e/ou pés. Ele é divido em três colunas, que apresentam as seguintes questões: 1) Considerando os últimos 12 meses, você tem tido algum problema (tal como dor, desconforto ou dormência) nas seguintes regiões? 2) Você tem tido algum problema, nos últimos 7 dias, nas seguintes regiões? 3) Durante os últimos 12 meses, você teve que evitar suas atividades normais (trabalho, serviço doméstico ou passatempo) por causa de problemas nas seguintes regiões?

Avaliou-se a força muscular lombar através do dinamômetro lombar da marca Takei ${ }^{\circledR}$, com escala de medida 0 a 200 quilograma-força (kgf). Para tanto, as pesquisadas ficaram em posição ortostática sobre a base do dinamômetro, com os pés apoiados no equipamento, joelhos estendidos, tronco flexionado e com ambas as mãos segurando o bastão. Um antebraço estava na posição supinada e o outro na posição pronada. Em seguida, deu-se a ordem para que iniciassem puxando o bastão para cima, utilizando a força da musculatura lombar para a extensão do tronco. Na dinamometria lombar, alguns comandos verbais precisaram ser utilizados, tais como: "posiciona!, pronto!, puxe! e força!”.

Realizou-se o teste três vezes e elegeu-se sempre o maior valor obtido em cada dinamometria para cada participante. Por último, aplicou-se um questionário retroativo de disfunções lombares contendo 20 perguntas, em que a resposta se firmava em opções de 1 a 5 (1: sem dor; 2: pouca dor; 3: dor moderada; 4: dor forte; 5: dor intensa). Após a coleta, tabularam-se os dados no programa Statistical Package for the Social Sciences - SPSS, versão 22.0, com a consequente análise.

Houve esclarecimento do estudo para as participantes quanto aos objetivos e aos métodos da pesquisa, ocasião em que se apresentou o convite para a participação e solicitou-se a assinatura do Termo de Consentimento Livre e Esclarecido (TCLE). O convite dos voluntários e a obtenção dos dados ocorreram após a aprovação do projeto no Comitê de Ética e Pesquisa (CEP) da UNESC, sob o Parecer n ${ }^{\circ} 1.993 .071$.

\section{RESULTADOS}

Ao todo, 130 participantes do sexo feminino integrantes do programa Ritmo e Saúde da AFASC compuseram a amostra da presente pesquisa. Ao invés de 110, ampliou-se a amostra para 130 participantes por conta da disponibilidade, da voluntariedade e da curiosidade em relação à pesquisa.

A média de idade fixou-se em 48,82 anos, com desvio padrão de $\pm 8,44$ anos. A idade mínima foi de 31 anos e a máxima de 60 anos. A dinamometria lombar apresentou como resultado de menor expressão 11 kgf e valor máximo de 91 kgf, gerando uma média de 54,35, com desvio padrão de $\pm 16,32 \mathrm{kgf}$. Esses resultados individuais, quando comparados com a idade e o sexo na tabela de referência, apontaram que apenas $16,9 \%(\mathrm{n}=10)$ das 130 participantes apresentaram nível normal de força. Portanto, $83,1 \%(n=120)$ das participantes alcançaram valores abaixo da normalidade, denotando fraqueza muscular. 
Os escores médios da escala visual analógica (EVA) encontrados são de 2, 99, com desvio padrão de $\pm 2,79$, em que o valor mínimo encontrado é 0 e o máximo é 10 na escala. Dividindo a EVA em faixas de intensidade, pode-se constatar na Tabela I que a maioria das participantes apresenta dor de intensidade mínima, seguida pela moderada e a intensa.

Os resultados apresentados pelo questionário retroativo de função e dor lombar apresentaram maior percentual envolvendo as questões 12,17 e 20, como se observa na Tabela II.

Tabela I - Escala visual analógica de mulheres com lombalgia crônica inespecífica e praticantes de um programa público de exercícios físicos em Criciúma, Santa Catarina, 2017.

\begin{tabular}{lc}
\hline Variáveis & $\mathbf{n}(\mathbf{\%})$ \\
$\mathbf{n}=\mathbf{1 3 0}$
\end{tabular}

EVA: escala visual analógica, n: número de pesquisados.

Tabela II - Distribuição quanto às características da dor e seus impactos funcionais pregressos nas mulheres com lombalgia crônica inespecífica e praticantes de um programa público de exercícios físicos em Criciúma, Santa Catarina, 2017.

\begin{tabular}{lccccc}
\hline & & \multicolumn{2}{c}{ Intensidade da dor (\%) } \\
& Nenhuma & Pouca & Moderada & Forte & Insuportável \\
\hline Q12 & $53,1 \%$ & $12,3 \%$ & $22,3 \%$ & $11,5 \%$ & $0,8 \%$ \\
Q17 & $50,0 \%$ & $13,8 \%$ & $20,8 \%$ & $13,1 \%$ & $2,3 \%$ \\
Q20 & $80,0 \%$ & $11,5 \%$ & $4,6 \%$ & $3,9 \%$ & $0,0 \%$ \\
\hline
\end{tabular}

Legenda: Respostas ao Questionário Retroativo de Função e Dor Lombar. Q12 - Se a dor aparecia durante o desempenho das funções no trabalho; Q17 - Se sentia dor no final do dia; Q20 - Se havia dor ao repousar.

Os resultados apresentados pelo ODQ indicam que: $97,7 \%(\mathrm{n}=127)$ das participantes apresentaram grau de incapacidade funcional mínimo; 2,3\% ( $\mathrm{n}=3)$ das participantes apresentam grau de incapacidade funcional moderado. Nenhuma das participantes apresenta grau de incapacidade funcional severo no momento (Tabela III). Ao se comparar o ODQ ao QNSO, identificou-se que, nos últimos 12 meses, 51,5\% $(\mathrm{n}=67)$ das mulheres apresentaram algum tipo de dor na região lombar e $16,9 \%$ $(\mathrm{n}=22)$ delas se afastaram de seus afazeres por conta da dor.

Tabela III - Oswestry Low Back Pain Disability Questionnaire (ODQ) 2.O / Questionário Nórdico de Sintomas Osteomusculares (QNSO) de mulheres com lombalgia crônica inespecífica e praticantes de um programa público de exercícios físicos em Criciúma, Santa Catarina, 2017.

\begin{tabular}{lc}
\hline Variáveis & $\begin{array}{c}\text { Percentual } \\
\text { (n= 130) }\end{array}$ \\
\hline ODQ 2.0 & $97,7 \%$ \\
Mínima & $2,3 \%$ \\
Moderada & $0,0 \%$ \\
Severa & \\
QNSO & $51,5 \%{ }^{*}$ \\
Dor nos últimos 12 meses & $30,0 \%{ }^{*}$ \\
Dor nos últimos 7 dias & $16,9 \%{ }^{*}$ \\
Afastamento atividades últimos 12 meses & \\
\hline
\end{tabular}

Legenda: *Os valores distribuem o percentual da totalidade por tópico independentemente explorado, ou seja, percentual que apresentou quadro de dor nos últimos 12 meses, o percentual que apresentou quadro doloroso lombar nos últimos 7 dias, bem como, o percentual que contou com afastamento das atividades nos últimos 12 dias. 


\section{DISCUSSÃO}

A lombalgia é um transtorno musculoesquelético bastante frequente, com potencial de levar à incapacidade funcional por seu forte impacto na qualidade de vida, nos níveis sociais de participação e de atividade ${ }^{(21)}$, motivo pelo qual é objeto de investigação.

A dor é uma experiência multidimensional, complexa, individual e subjetiva, sendo que a sua cronificação ${ }^{(22)}$, embora apresente tendência de redução da intensidade (conforme se observou no presente estudo), pode gerar adaptações físicofuncionais, como a fraqueza da musculatura envolvida no segmento vertebral lombar.

Neste sentido, os resultados encontrados, de 93,1\% das voluntárias da atual pesquisa com intensidades dolorosas lombares entre mínima e moderada, apresentam-se dissociados das funções ocupacionais e do horário do dia. Esses achados, associados ao de $83 \%$ de voluntárias com redução significativa da força muscular, contribuem para caracterizar esses casos de lombalgia cronificada.

Ao considerar o tempo em que a dor persiste, ou seja, a sua duração, é possível classificá-la como aguda, subaguda ou crônica, caracterizando seu estagiamento. A dor aguda está associada à lesão. Tem curta duração e tende a sumir com a cicatrização da lesão. Já a dor crônica é persistente, recorrente e não está necessariamente associada a uma lesão no organismo. A cronificação da dor pode ser de causa desconhecida. Em sua classificação, consideram-se crônicas aquelas que permanecem por mais de três meses, caso das síndromes dolorosas, como lombalgias crônicas inespecíficas ${ }^{(23)}$.

No presente estudo, percebeu-se que todas as participantes sentiram dor na região lombar em algum momento de sua vida, podendo a dor estar associada ao sedentarismo, à má postura no ambiente de trabalho, à fraqueza muscular na região lombar ou à execução errônea nos exercícios prescritos no programa.

Um estudo realizado sobre a lombalgia que buscou desmistificar a sua origem gerou a hipótese de que a dor lombar crônica inespecífica pode estar diretamente ligada ao não recrutamento, ou menor recrutamento, das fibras dos músculos estabilizadores da coluna lombar, como o músculo transverso do abdômen, e pelas fibras profundas do músculo multifídeo. Com isto, o seu tratamento torna-se complexo, não se limitando apenas ao fortalecimento dos músculos lombares. Pelo contrário, deve-se pensar no paciente como um todo ${ }^{(24)}$.

Em um estudo transversal com objetivo de avaliar os sintomas osteomusculares em adultos ativos e sedentários, com 49 participantes, todos acima de 47 anos e de ambos os sexos, predominando o feminino, deparou-se com 29 indivíduos ativos e com 20 sedentários. O resultado encontrado é que, nos últimos doze meses, os ativos apresentaram 10\% de queixas dolorosas e os sedentários, 45\%. Já nos últimos sete dias, as queixas dolorosas são de 7\% nos ativos e de 40\% nos sedentários. Isso revela que as dores crônicas tornam-se mais comuns nos indivíduos sedentários ${ }^{(25)}$.

Uma pesquisa relata que a diminuição da força muscular pode remeter à redução da autonomia na realização de exercícios ou atividades de vida diária (AVDs). Além disso, o déficit muscular pode ter relação com limitação funcional, morbidade e mortalidade independente da faixa etária. Logo, a quantificação da força muscular é indispensável para comparação de outros fatores relacionados à lombalgia ${ }^{(26,27)}$.

Apesar de a lombalgia crônica inespecífica estar presente nas praticantes voluntárias do presente estudo, a incapacidade encontrada é considerada baixa, mostrando-se desconectada da presença de dor. A manutenção de atividade em um programa comunitário com diversificações de tipos de exercícios contribui para essa resposta, bem como os níveis de intensidade dolorosa baixos.

Um estudo separou em dois grupos 40 mulheres que possuíam dor lombar crônica, com intervalo de idade entre 30 e 50 anos, em que um deles realizou exercícios para estabilização segmentar lombar e o outro realizou esses mesmos exercícios associados ao fortalecimento de glúteos durante seis semanas. Observou-se um efeito mais expressivo nos indivíduos que realizaram os exercícios de estabilização segmentar lombar associado ao fortalecimento de glúteos ${ }^{(21,28)}$.

A ausência da prática de atividade física é uma variável que pode demonstrar associação com incapacidade. Trabalhos que avaliaram indivíduos com dor lombar identificaram que a dor está associada a menores níveis de atividade física ${ }^{(28,29)}$. Da mesma forma, outra pesquisa apresentou relação entre incapacidade e nível de atividade aeróbica em pacientes com lombalgia crônica, em que a lombalgia mostrou-se reduzida nesses indivíduos em relação aos controles ${ }^{(30)}$. Outro estudo evidenciou que o exercício aeróbico moderado realizado três vezes por semana por pessoas com lombalgia crônica inespecífica é capaz de reduzir o quadro doloroso, reduzir mediadores bioquímicos relacionados à fisiopatologia da dor musculoesquelética, como a creatina-quinase, e parâmetros de estresse oxidativo ${ }^{(23)}$.

Um estudo de revisão mostrou que o exercício tem efeitos redutivos na dor lombar e, como consequência, gera aumento dos níveis de funcionalidade. Exercícios que visam à resistência, ao fortalecimento muscular, ao controle postural e aos alongamentos podem servir não só como tratamento proposto em quadros lombálgicos, mas também como forma de prevenção. Tal prática pode ser realizada de maneira individual ou em grupo ${ }^{(31)}$.

Boa parte dos efeitos induzidos pelo exercício físico, como o aumento da massa muscular, a melhora do sistema cardiovascular, a redução da incidência de doenças e a liberação de opióides endógenos, como as beta-endorfinas, devem-se principalmente às adaptações induzidas sobre os diversos sistemas corporais de melhoria da sua eficiência fisiológica ${ }^{(32)}$. 
Da mesma forma, outra pesquisa apresentou relação entre incapacidade e nível de atividade aeróbica em pacientes com lombalgia crônica, esta mostrando-se reduzida nestes indivíduos em relação aos controles ${ }^{(30)}$.

Há de se considerar que por muito tempo o repouso foi preconizado para casos de lombalgia crônica, mas sabe-se mais recentemente que o exercício físico praticado com regularidade diminui quadros de dor lombar ${ }^{(23)}$.

Entre as limitações do presente estudo, encontra-se a carência de informações pré-programa das participantes, destacando a importância do registro de informações gerais de saúde quando do início de programas comunitários.

\section{CONCLUSÃO}

O programa possui características que contribuem para manter controlada a intensidade dolorosa, que vem se mostrando baixa, bem como a incapacidade funcional significativa dos participantes. A fraqueza muscular na região lombar, apresentada pelas participantes sem associação com a funcionalidade, e a dor podem ser um fator que perpetua os quadros de lombalgia crônica. Os achados sugerem a necessidade de revisão da prescrição terapêutica dos exercícios do programa realizado.

\section{CONFLITOS DE INTERESSE}

Não há conflito de interesses envolvendo este estudo.

\section{REFERÊNCIAS}

1. Organização Pan-Americana da Saúde, Organização Mundial da Saúde. A saúde no Brasil. Brasília: OPAS/OMS; 1998.

2. Lowy A, Bernhard J. Quantitative assessment of changes in patients constructs of quality of life: An application of multilevel models. Qual Life Res. 2004;13(7):1177-85.

3. Conselho Regional de Fisioterapia e Terapia Ocupacional (BR). Política Nacional de Saúde Funcional: construindo mais saúde para a população [Internet]. Brasília: CREFITO; 2011 [acesso em 2016 Ago 20]. Disponível em: http://www. crefito8.org.br/site/servicos/pnsf_07_01_12.pdf

4. Gazzineli MF, Gazzinelli A, Reis DC, Penna CMM. Educação em saúde: conhecimentos, representações sociais e experiência da doença. Cad Saúde Publica. 2005;21(1):200-6.

5. Augusto VG, Aquino CF, Machado NC, Cardoso VA, Ribeiro S. Promoção de saúde em unidades básicas: análise das representações sociais dos usuários sobre a atuação da fisioterapia. Ciênc Saúde Coletiva. 2011;16(Supl 1):957-63.

6. Lee IM, Shiroma EJ, Lobelo F, Puska P, Blair SN, Katzmarzyk PT. Effect of physical inactivity on major non-communicable diseases worldwide: an analysis of burden of disease and life expectancy. The Lancet. 2012;380(9838):219-29.

7. Furtado RNV, Ribeiro LH, Abdo BA, Descio SJ, Martucci CE Junior, Serruya DC. Dor lombar inespecífica em adultos jovens: fatores de risco associados. Rev Bras Reumatol. 2014;54(5):371-7.

8. Valença JB, Ferraz KP, Alencar MCB, Souza FG, Lopes LV. Perfil de trabalhadores com doenças da coluna vertebral atendidos em um serviço de saúde. Cad Ter Ocup UFSCAR. 2016;24(2):227-33.

9. Marcelo LM, Martins MS, Longen WC. Avaliação da funcionalidade e da força dinamométrica lombar de mineiros do carvão. Rev Inova Saúde. 2015;4(2):115-27.

10. Barreto RR. Perfil dos Pacientes com Lombalgia Crônica da Clinica Escola da UEG - ESEFFEGO. In: III Congresso de Ensino, Pesquisa e Extensão da UEG. Pirenópolis, 2016 Nov 19, 20 e 21.

11. Rasmussen CD, Jorgensen MB, Clausen T, Andersen LL, Stroyer J, Holtermann A. Does self-assessed physical capacity predict development of low back pain among health care workers? A 2-year follow-up study. Spine. 2013;38(3):272-6.

12. Bell JA, Burnett A. Exercise for the primary, secondary and tertiary prevention of low back pain in the workplace: a systematic review. J Occup Rehabil. 2009;19(1):8-24

13. Graupe S, Bergmann MLA, Bergmann GG. Prevalência de dor lombar inespecífica e fatores associados em adolescentes de Uruguaiana/RS. Rev Bras Ortop. 2014;49(6):661-7.

14. Silva MR, Ferretti F, Lutinski JA. Dor lombar, flexibilidade muscular e relação com o nível de atividade física de trabalhadores rurais. Saúde Debate. 2017;41(112):183-94.

15. Lizier DT, Perez MV, Sakata RK. Exercícios para Tratamento de Lombalgia Inespecífica. Rev Bras Anestesiol. 2012;62(6):838-46. 
16. Ferreira ML, Ferreira PH, Latimer J, Herbert RD, Hodges PW, Jennings MD, et al. Comparison of general exercise, motor control exercise and spinal manipulative therapy for chronic low back pain: a randomized trial. Pain. 2007;131(1-2);31-7.

17. Ostelo RW, Tulder MW, Vlaeyen JW. Behavioural treatment for chronic low-back pain. 2005.

18. Rainville J, Nguyen R, Suri P. Effective conservative treatment for chronic low back pain. Semin Spine Surg. 2009;21(4):25763.

19. Martins NS. Adaptação cultural e linguística do Oswestry Low Back Pain Disability Questionarie - ODQ 2.0. Coimbra: Escola Superior de Tecnologia da Saúde de Coimbra; 2002.

20. Saime AY, Hatice EK, Deniz E, Sibel K. Efetividade do kinesio taping na dor e incapacidade na síndrome dolorosa miofascial cervical. Rev Bras Reumatol. 2017;57(2):93-9.

21. Wagner MP. Influência da estabilização segmentar core na dor e funcionalidade da coluna lombar. Fisioter Brasil. 2017;18(2):148-53.

22. Sousa FF, Silva JA. The metric of pain: theoretical and methodological issues. Rev Dor. 2005;6(1):469-513.

23. Longen WC. Efeitos do exercício aeróbico e da terapia manual sobre marcadores bioquímicos de lesão musculoesquelética e parâmetros funcionais em motoristas profissionais com lombalgia crônica inespecífica [tese]. Criciúma: UNESC; 2013.

24. Ferreira LM. Associação entre recrutamento de músculos abdominais com desfechos clínicos e risco prognóstico em indivíduos com dor lombar crônica inespecífica: um estudo preliminar. Fisioter Pesqui. 2016:23(1):45-51.

25. Campos LP, Reichert FF. Prevalência de sintomas osteomusculares em indivíduos ativos e sedentários. Fisioter Brasil. 2012;13(3):189-93.

26. Bittencourt SW, Nasrala E Neto. Correlação entre lombalgia e capacidade funcional em idosos. Rev Bras Geriatr Gerontol. 2016;19(6):987-94.

27. Domenech SC, Gomes NB. Dinamometria lombar: um teste funcional para o tronco. Rev Bras Med Trab. 2016; 14(2): $120-6$.

28. Rafaelli CS, Argentino PJ. Prevalência de dor lombar em praticantes de musculação. Rev UNIFEBE. 2010; 1(8):190-8.

29. Dijken CB, Fjellman-Wiklund A, Hildingsson C. Low back pain, lifestyle factors and physical activity: a population-based study. J Rehabil Med. 2008;40(10):864-9.

30. Smeets RJ, Wittink H, Hidding A, Knottnerus JA. Do patients with chronic low back pain have a lower level of aerobic fitness than healthy controls?: are pain, disability, fear of injury, working status, or level of leisure time activity associated with the difference in aerobic fitness level? Spine. 2006;31(1):90-7.

31. Jeong UC, Sim JH, Kim CY, Hwang-Bo G, Nam CW. The effects of gluteus muscle strengthening exercise and lumbar stabilization exercise on lumbar muscle strength and balance in chronic low back pain patients. J Phys Ther Sci 2015;27(12):3813-6.

32. Steiger F, Wirth B, Bruin ED, Mannion F. Is a positive clinical outcome after exercise therapy for chronic non-specific low back pain contingent upon a corresponding improvement in the targeted aspect(s) of performance? A systematic review. Eur Spine J. 2012;21(4):575-98.

\section{Endereço primeiro autor:}

Maicon da Silva Martins

Universidade do Extremo Sul Catarinense - UNESC

Programa de Pós-Graduação em Saúde Coletiva

Rua Celestina Zilli Rovaris, 97/106

Bairro: Centro

CEP: 88.802-210 - Criciúma - SC - Brasil

E-mail: maicom.martins@hotmail.com

\section{Endereço para correspondência:}

Willians Cassiano Longen

Universidade do Extremo Sul Catarinense - UNESC

Programa de Pós-Graduação em Saúde Coletiva

Avenida Universitária, 1105

Bairro: Universitário

CEP: 88.806-000 - Criciúma - SC - Brasil

E-mail:wcl@unesc.net 